\title{
Point source estimation via finite element multiple-model Kalman filtering
}

\author{
G. Battistelli, L. Chisci, N. Forti, G. Pelosi, S. Selleri
}

\begin{abstract}
Point source estimation consists of detecting and localizing a concentrated diffusive source as well as estimating its intensity and induced field from pointwise-in-time-and-space measurements of sensors deployed over the area of interest. The spatiotemporal dynamics of the diffused field is modeled by a partial differential equation (PDE) and a finite element (FE) method is employed for spatially discretizing the PDE model. Source identifiability, i.e. the possibility of detecting the source and uniquely identifying its location and intensity, is analysed in a system-theoretic framework. Further, a novel multiple model filtering approach to source estimation is presented and its effectiveness is demonstrated via a simulation experiment.
\end{abstract}

\section{INTRODUCTION}

The estimation of diffusive sources (e.g. of heat, polluting agents, toxic biochemical substances, etc.) has recently received great attention within both the signal processing and control communities for at least two reasons: i) the low-cost availability of wireless sensors measuring the induced field (e.g. temperature, concentration) which can be deployed at low cost and in large number over the area to be monitored; ii) the strategic importance of such a task in homeland security, environmental and industrial monitoring, military situation awareness for a wide range of applications (e.g. fire detection, pollution monitoring, detection and localization of terrorist biochemical attacks, etc.). By source estimation, in this paper, we refer to the joint task of detecting its presence, localizing it, estimating its intensity and monitoring the induced field. To this end, two mainstream approaches can be found in the literature. A first approach [1]-[3] models the source-induced field in steady-state, thus disregarding its transient time evolution, and therefore yields a parametric (static) estimation problem. It is worth to point out that, for slowly diffusing sources, this can imply a very long, possibly unacceptable, detection/localization delay. Conversely, the second approach [4]-[7] is to explicitly take into account the spatiotemporal diffusion dynamics thus yielding a state (dynamic) estimation problem.

In order to allow faster detection/localization of slowly diffusing sources, this paper will follow the latter, dynamic, approach. In particular, the spatiotemporal diffusion dynamics is modelled by an advection-diffusion partial differential equation (PDE) with appropriate boundary conditions and a point (concentrated) source is considered. The finite

\footnotetext{
The authors are with Dipartimento di Ingegneria dell'Informazione, Università degli Studi di Firenze, Via Santa Marta 3, 50139 Firenze, Italy \{giorgio.battistelli, luigi.chisci, nicola.forti, giuseppe.pelosi, stefano.selleri\}eunifi.it
}

element (FE) method is exploited for spatial discretization of the PDE. After time-discretization, the original infinite-dimensional boundary value problem is, therefore, transformed into a finite-dimensional, possibly large-scale, discrete-time linear system with state vector consisting of the field values in the vertices of the FE mesh, input vector representing the source intensity and input matrix depending on the source location. In this framework, this paper provides two major contributions to the source estimation problem. First, inspired by the classic notion of structural identifiability [8]-[9] considered as an a priori analysis for experiment design [9], this work defines the concept of source identifiability, i.e. the possibility of detecting the source and uniquely determining its position and intensity from available pointwise-in-time-and-space field measurements.

Specifically, system-theoretic conditions for identifiability are derived in terms of rank tests on suitable polynomial matrices for both cases in which the source intensity is regarded as an unknown input or is modeled as the output of an appropriate exosystem. Then, a multiple-model Kalman filtering approach to source estimation is undertaken by considering all hypotheses (modes) corresponding to the source location in any possible element of the FE mesh plus a further hypothesis accounting for the possible source absence. Both cases of motionless source with unknown position and of moving source are addressed, resorting to the static Multiple Model (MM) and, respectively, dynamic Interacting Multiple Model (IMM) algorithms. It is worth pointing out that the source estimation/identification problem has been also addressed in [10] as an inverse PDE problem, while in the present work it is regarded as a hybrid state estimation problem making use of space discretization.

\section{PROBLEM FORMULATION}

Let us consider a diffusion process governed by a PDE of the form

$$
\frac{\partial x}{\partial t}+\mathcal{A}(x)=f \text { in } \Omega
$$

with possibly inhomogeneous boundary condition

$$
\mathcal{B}(x)=g \quad \text { on } \partial \Omega .
$$

where: $x(\mathbf{p}, t)$ is the space-time dependent scalar field of interest (e.g. concentration, temperature), defined over the space-time domain $\Omega \times \mathbb{R}$; the space domain $\Omega$ is supposed to be bounded and with smooth boundary $\partial \Omega ; \mathbf{p} \in \Omega$ denotes the $d$-dimensional $(d \in\{1,2,3\})$ position vector; $t \in \mathbb{R}$ denotes time; $\mathcal{A}(\cdot)$ and $\mathcal{B}(\cdot)$ are the advection-diffusion and, respectively, Robin operators defined as $\mathcal{A}(x)=-\lambda \nabla^{2} x+$ 
$\mathbf{v}^{T} \nabla x$ and $\mathcal{B}(x)=\partial x / \partial \mathbf{n}+\beta x . \lambda$ is a constant diffusion coefficient; $\mathbf{v}(\mathbf{p})$ is the advection velocity vector; $\beta(\mathbf{p}) \geq 0$ is a, possibly space-dependent, coefficient; $\partial x / \partial \mathbf{n}=\mathbf{n}^{T} \nabla x$, $\mathbf{n}$ being the outward pointing unit normal vector of the boundary $\partial \Omega ; g(\mathbf{p}, t)$ is the forcing term acting on the boundary $\partial \Omega$.

The term $f(\mathbf{p}, t)$ is the point source input modeled as $f(\mathbf{p}, t)=0$ if no source exists, or $f(\mathbf{p}, t)=$ $u(t) \delta\left(\mathbf{p}-\mathbf{p}^{0}(t)\right)$ otherwise, with unknown intensity $u(t)$ and position $\mathbf{p}^{0}(t) \in \Omega$. The aim is to detect the source presence and jointly estimate $u(t), \mathbf{p}^{0}(t), x(\mathbf{p}, t)$ given measurements

$$
y_{k, i}=h_{i}\left(x\left(\mathbf{s}_{i}, t_{k}\right)\right)+v_{k, i}
$$

provided by sensors $i \in \mathcal{S} \triangleq\{1, \ldots, S\}$, located at positions $\mathbf{s}_{i} \in \Omega$, at discrete sampling instants $t_{k}, k \in \mathbb{Z}_{+}=$ $\{1,2, \ldots\}$, such that $0<t_{1}<t_{2}<\cdots$.

The above stated dynamic estimation problem is clearly infinite-dimensional. It will be shown in the next section how it can be approximated into a finite-dimensional one by exploiting the finite element (FE) method.

\section{FINITE ELEMENT APPROXIMATION}

The PDE (1) with boundary condition (2) can be recast into the following integral form:

$\int_{\Omega} \frac{\partial x}{\partial t} \varphi d \mathbf{p}-\lambda \int_{\Omega} \nabla^{2} x \varphi d \mathbf{p}+\int_{\Omega} \mathbf{v}^{T} \nabla x \varphi d \mathbf{p}=\int_{\Omega} f \varphi d \mathbf{p}$

where $\varphi(\mathbf{p})$ is a generic space-dependent weight function. By applying Green's identity and thanks to (2), one obtains:

$$
\begin{aligned}
\int_{\Omega} \frac{\partial x}{\partial t} \varphi d \mathbf{p}+\lambda \int_{\Omega} \nabla^{T} x \nabla \varphi d \mathbf{p}+\int_{\Omega} \mathbf{v}^{T} \nabla x \varphi d \mathbf{p} \\
-\lambda \int_{\partial \Omega}(g-\beta x) \varphi d \mathbf{p}=\int_{\Omega} f \varphi d \mathbf{p}
\end{aligned}
$$

By subdividing the domain $\Omega$ into a suitable set of non overlapping elements and by defining a suitable set of basis functions $\phi_{j}(\mathbf{p}), j=1, \ldots, n$, on them, it is possible to write an approximation of the unknown function $x(\mathbf{p}, t)$ as

$$
x(\mathbf{p}, t)=\sum_{j=1}^{n} \phi_{j}(\mathbf{p}) x_{j}(t)=\phi^{T}(\mathbf{p}) \mathbf{x}(t)
$$

where: $x_{j}(t)$ is the unknown expansion coefficient of function $x(\mathbf{p}, t)$ relative to time $t$ and basis function $\phi_{j}(\mathbf{p})$; $\phi(\mathbf{p}) \triangleq \operatorname{col}\left\{\phi_{j}(\mathbf{p})\right\}_{j=1}^{n}$ and $\mathbf{x}(t) \triangleq \operatorname{col}\left\{x_{j}(t)\right\}_{j=1}^{n}$.

The choices of the basis functions $\phi_{j}$ and of the elements are key points of the FE method. Typically, the elements (triangles or quadrilaterals in 2D, polyhedral in 3D) define a FE mesh with vertices $\mathbf{p}_{j} \in \Omega, j=1, \ldots, n$. Then each basis function $\phi_{j}$ is a piece-wise polynomial which vanishes outside the FEs around $\mathbf{p}_{j}$ and such that $\phi_{j}\left(\mathbf{p}_{i}\right)=\delta_{i j}, \delta_{i j}$ denoting the Kronecker delta.

By choosing the test function $\varphi$ equal to the selected basis functions, the Galerkin weighted residual method is applied and the following equation is obtained [11]-[12]

$$
\begin{aligned}
& \underbrace{\left[\int_{\Omega} \phi(\mathbf{p}) \boldsymbol{\phi}^{T}(\mathbf{p}) d \mathbf{p}\right]}_{\mathbf{M}} \dot{\mathbf{x}}(t)+\underbrace{\left[\lambda \int_{\Omega} \nabla \phi(\mathbf{p}) \nabla \phi^{T}(\mathbf{p}) d \mathbf{p}\right]}_{\mathbf{S}} \mathbf{x}(t) \\
& +\underbrace{\left[\int_{\Omega} \boldsymbol{\phi}(\mathbf{p}) \mathbf{v}^{T}(\mathbf{p}) \nabla \phi^{T}(\mathbf{p}) d \mathbf{p}\right]}_{\mathbf{G}} \mathbf{x}(t) \\
& +\underbrace{\left[\lambda \int_{\partial \Omega} \beta(\mathbf{p}) \phi(\mathbf{p}) \phi^{T}(\mathbf{p}) d \mathbf{p}\right]}_{\mathbf{Q}_{\beta}} \mathbf{x}(t)= \\
& =\underbrace{\left[\int_{\Omega} \boldsymbol{\phi}(\mathbf{p}) \delta\left(\mathbf{p}-\mathbf{p}^{0}\right) d \mathbf{p}\right]}_{\phi\left(\mathbf{p}^{0}\right)} u(t)+\underbrace{\left[\lambda \int_{\partial \Omega} \boldsymbol{\phi}(\mathbf{p}) \phi^{T}(\mathbf{p}) d \mathbf{p}\right]}_{\mathbf{Q}_{1}} \mathbf{g}(t)
\end{aligned}
$$

where in the integrals on the contour $\partial \Omega$ it is assumed that the various functions are the restrictions to $\partial \Omega$ of the original functions defined over $\Omega$, and that for $g(\mathbf{p}, t)$ an expansion akin to (4) holds, i.e. $g(\mathbf{p}, t)=$ $\sum_{j=1}^{n} \phi_{j}(\mathbf{p}) g_{j}(t)=\phi^{T}(\mathbf{p}) \mathbf{g}(t)$.

It is evident how all integrals in the LHS (5) depend only on basis functions and can be computed a priori. In particular, the first two integrals yields the well known mass and stiffness matrices $\mathbf{M}$ and $\mathbf{S}$ [11]. Matrices $\mathbf{G}, \mathbf{Q}_{\beta}$ and $\mathbf{Q}_{1}$ are non standard but can be easily computed in simplex coordinates for first order triangular elements [11], [13].

Then, by regularly discretizing in time (5) with sampling interval $\delta t$ (i.e. $t_{k}=k \delta t$ ) and approximating the time derivative with the finite difference $\dot{\mathbf{x}}(t) \simeq\left(\mathbf{x}_{k+1}-\mathbf{x}_{k}\right) / \delta t$, the following discrete-time linear descriptor system is obtained:

$$
\begin{aligned}
& \mathbf{M}\left(\frac{\mathbf{x}_{k+1}-\mathbf{x}_{k}}{\delta t}\right)+\mathbf{S} \mathbf{x}_{k+1} \\
& \quad+\mathbf{G x}_{k+1}+\mathbf{Q}_{\beta} \mathbf{x}_{k+1} \simeq \boldsymbol{\phi}\left(\mathbf{p}^{0}\right) u_{k}+\mathbf{Q}_{1} \mathbf{g}_{k+1}
\end{aligned}
$$

from which one obtains the discrete-time model

$$
\mathbf{x}_{k+1}=\mathbf{A} \mathbf{x}_{k}+\mathbf{B}\left(\mathbf{p}^{0}\right) u_{k}+\mathbf{b}_{k}+\mathbf{w}_{k}
$$

where $u_{k}=u\left(t_{k+1}\right), \mathbf{w}_{k}$ is a process disturbance taking into account also the space-time discretization errors, and

$$
\begin{aligned}
\mathbf{A} & =\left[\mathbf{I}+\delta t \mathbf{M}^{-1}\left(\mathbf{S}+\mathbf{G}+\mathbf{Q}_{\beta}\right)\right]^{-1} \\
\mathbf{B}\left(\mathbf{p}^{0}\right) & =\left[\mathbf{I}+\delta t \mathbf{M}^{-1}\left(\mathbf{S}+\mathbf{G}+\mathbf{Q}_{\beta}\right)\right]^{-1} \mathbf{M}^{-1} \delta t \boldsymbol{\phi}\left(\mathbf{p}^{0}\right) \\
\mathbf{b}_{k} & =\left[\mathbf{I}+\delta t \mathbf{M}^{-1}\left(\mathbf{S}+\mathbf{G}+\mathbf{Q}_{\beta}\right)\right]^{-1} \mathbf{M}^{-1} \delta t \mathbf{Q}_{1} \mathbf{g}_{k+1}
\end{aligned}
$$

For a quantitative characterization of such errors, the reader can refer to [14]. Notice that, in equation (7), the intensity $u_{k}$ and the position $\mathbf{p}^{0}$ of the point source input are unknown and hence must be estimated together with the state vector $\mathbf{x}_{k}$. As for the intensity $u_{k}$, different models are possible:

(a) $u_{k}$ is treated as an unknown input for which no information on the possible time evolution is available [15], [16];

(b) $u_{k}$ is unknown but a dynamic model for its time evolution is available, i.e., it is supposed that $u_{k}$ is generated as the output of an auxiliary linear system (called exosystem).

$$
\begin{aligned}
\mathbf{q}_{k+1} & =\mathbf{F} \mathbf{q}_{k}+\boldsymbol{\zeta}_{k} \\
u_{k} & =\mathbf{H} \mathbf{q}_{k}
\end{aligned}
$$


where $\mathbf{q}_{k}$ is the exosystem state and $\boldsymbol{\zeta}_{k}$ the disturbance input. Here, without loss of generality the pair $(\mathbf{F}, \mathbf{H})$ is supposed to be observable.

For instance, if it is known that the unknown intensity $u_{k}$ can vary slowly with time, its time evolution can be modeled as a random walk by letting $\mathbf{F}=\mathbf{H}=1$ and taking $\boldsymbol{\zeta}_{k}$ as a zeromean white noise. Of course, the preferable model depends on the situation under consideration and, specifically, on possible physical insights on the source intensity.

\section{SOURCE IDENTIFIABILITY}

In this section, an analysis on the possibility of correctly identifying the unknown source location $\mathbf{p}^{0}$ and intensity $u_{k}$ is provided both in cases (a) and (b). As usually done in observability/identifiability analysis, the study is carried out in the ideal noise-free case by supposing that the measurements $\mathbf{y}_{k}$ are generated by

$$
\begin{aligned}
\mathbf{x}_{k+1} & =\mathbf{A} \mathbf{x}_{k}+\mathbf{B}\left(\mathbf{p}^{0}\right) u_{k} \\
\mathbf{y}_{k} & =\mathbf{C} \mathbf{x}_{k}
\end{aligned}
$$

Accordingly, in case (b), the intensity dynamics will be supposed to be noise-free by letting

$$
\begin{aligned}
\mathbf{q}_{k+1} & =\mathbf{F} \mathbf{q}_{k} \\
u_{k} & =\mathbf{H} \mathbf{q}_{k}
\end{aligned}
$$

Notice that the known input $\mathbf{b}_{k}$ is not considered in equation (8) since, thanks to the superposition principle for linear systems, its contribution is immaterial to the source identification problem.

Let now $\mathbb{S}$ be the linear space of all real-valued sequences on the nonnegative integers $\mathbb{Z}_{+}$and let us denote by $\mathcal{U} \subseteq \mathbb{S}$ the set of all possible time-evolutions of the intensity $u_{k}$ which are consistent with the available model. Clearly, in case (a) we simply have $\mathcal{U}=\mathbb{S}$, while in case (b) $\mathcal{U}$ is the set of all the possible output behaviors of system (9). Let also denote by $\mathbf{y}\left(\mathbf{x}_{0}, \mathbf{p}^{0}, u\right)$ the output behavior of system (8) when the initial state is $\mathbf{x}_{0}$, the source location is $\mathbf{p}^{0}$ and the source intensity evolves according to the sequence $u$. The following notion can be introduced.

Definition 1: The point source is said to be identifiable if $\mathbf{y}\left(\mathbf{x}_{0}, \mathbf{p}^{0}, u\right) \neq \mathbf{y}\left(\overline{\mathbf{x}}_{0}, \overline{\mathbf{p}}^{0}, \bar{u}\right)$ for any pair of source locations $\mathbf{p}^{0}, \overline{\mathbf{p}}^{0} \in \Omega$, any pair of initial states $\mathbf{x}_{0}, \overline{\mathbf{x}}_{0}$, and any nonzero pair of intensity sequences $u, \bar{u} \in \mathcal{U}$, with $\left(\mathbf{p}^{0}, u\right) \neq\left(\overline{\mathbf{p}}^{0}, \bar{u}\right)$.

In words, identifiability of the point source corresponds to the fact that different sources (in terms of location and intensity) always give rise to different output behaviors or, equivalently, corresponds to the invertibility of the mapping from $\left(\mathbf{p}^{0}, u\right)$ to the output sequence $\mathbf{y}$. Notice that, in the above definition, we exclude the trivial case in which both $u$ and $\bar{u}$ are zero, but we allow that either $u$ or $\bar{u}$ be zero so as to account for the possibility of distinguishing between presence or absence of the source input.

Since only the observable part of (8) influences the output behavior $\mathbf{y}$, it is convenient to consider an alternative representation of system (8) obtained by means of the Kalman observability decomposition. This amounts to considering an invertible transformation matrix $\mathbf{T}$ such that

$$
\begin{aligned}
& \mathbf{T}^{-1} \mathbf{A} \mathbf{T}=\left[\begin{array}{cc}
\mathbf{A}_{11} & \mathbf{0} \\
\mathbf{A}_{21} & \mathbf{A}_{22}
\end{array}\right], \quad \mathbf{T}^{-1} \mathbf{B}=\left[\begin{array}{c}
\mathbf{B}_{1}\left(\mathbf{p}^{0}\right) \\
\mathbf{B}_{2}\left(\mathbf{p}^{0}\right)
\end{array}\right] \\
& \mathbf{C T}=\left[\begin{array}{ll}
\mathbf{C}_{1} & \mathbf{0}
\end{array}\right] .
\end{aligned}
$$

with $\left(\mathbf{A}_{11}, \mathbf{C}_{1}\right)$ observable. Of course, when $\mathbf{C}$ is chosen so that $(\mathbf{A}, \mathbf{C})$ is observable, we have $\mathbf{A}_{11}=\mathbf{A}$ and $\mathbf{C}_{1}=\mathbf{C}$. All the results of this section will refer to the system (8) or (8)-(9) in the case (b), under the observability decomposition (10). By exploiting standard results on observability and left invertibility of linear systems, the following theorem can now be stated.

Theorem 1: For any pair of distinct source locations $\mathbf{p}^{0}, \overline{\mathbf{p}}^{0} \in \Omega$, consider the polynomial matrix

$$
\boldsymbol{\Psi}\left(z, \mathbf{p}^{0}, \overline{\mathbf{p}}^{0}\right)=\left[\begin{array}{ccc}
z \mathbf{I}-\mathbf{A}_{11} & \mathbf{B}_{1}\left(\mathbf{p}^{0}\right) & \mathbf{B}_{1}\left(\overline{\mathbf{p}}^{0}\right) \\
\mathbf{C}_{1} & \mathbf{0} & \mathbf{0}
\end{array}\right]
$$

with $z \in \mathbb{C}$. Then, the following facts hold:

(i) in case (a), the source is identifiable if and only if

$$
\operatorname{rank}\left\{\boldsymbol{\Psi}\left(z, \mathbf{p}^{0}, \overline{\mathbf{p}}^{0}\right)\right\}=n_{o}+2 .
$$

for any $z \in \mathbb{C}$ and for any $\mathbf{p}^{0}, \overline{\mathbf{p}}^{0} \in \Omega$ with $\mathbf{p}^{0} \neq \overline{\mathbf{p}}^{0}$. Here $n_{o}$ is the dimension of the observable part of (8); (ii) in case (b), the source is identifiable if and only if the rank condition (11) holds for any $z \in \operatorname{sp}\{\mathbf{F}\}$ and for any $\mathbf{p}^{0}, \overline{\mathbf{p}}^{0} \in \Omega$ with $\mathbf{p}^{0} \neq \overline{\mathbf{p}}^{0}$. Here, $\operatorname{sp}\{\mathbf{F}\}$ stands for the spectrum of the matrix $\mathbf{F}$.

Notice that the only difference between cases (a) and (b) is that in the latter case only the values of $z$ corresponding to eigenvalues of the exosystem have to be considered in the rank test. Further, in this case, the identifiability condition can be rephrased in terms of system gains as follows.

Corollary 1: Consider case (b) and suppose that $\operatorname{sp}\{\mathbf{A}\}$ and $\operatorname{sp}\{\mathbf{F}\}$ are disjoint. Then, the source is identifiable if and only if

$$
\operatorname{rank}\left[\mathbf{C}(z \mathbf{I}-\mathbf{A})^{-1} \mathbf{B}\left(\mathbf{p}^{0}\right), \quad \mathbf{C}(z \mathbf{I}-\mathbf{A})^{-1} \mathbf{B}\left(\overline{\mathbf{p}}^{0}\right)\right]=2
$$

for any $z \in \operatorname{sp}\{\mathbf{F}\}$ and any $\mathbf{p}^{0}, \overline{\mathbf{p}}^{0} \in \Omega$ with $\mathbf{p}^{0} \neq \overline{\mathbf{p}}^{0}$.

From Theorem 1 and Corollary 1, it is evident that at least 2 sensors are needed in order to guarantee distinguishability of two source locations $\mathbf{p}^{0}, \overline{\mathbf{p}}^{0}$. However, this is just a lower bound since, in general, a larger number of sensors may be needed. The main drawback of the derived rank conditions is that they have to be satisfied for any pair of source locations $\mathbf{p}^{0}, \overline{\mathbf{p}}^{0}$ belonging to the space domain $\Omega$. This means that, in order to verify whether a given set of sensors ensures source identifiability, an infinite number of conditions have to be checked, which clearly makes the test impractical. A first, approximated, approach to sidestep such a difficulty would amount to restricting the attention only to a finite number of possible source locations, for example corresponding to the vertices $\mathbf{p}_{i}$ of the FE mesh. A second, more theoretically sound approach consists in looking for alternative conditions by exploiting the structure of the system matrices resulting from application of the FE method. 
With this respect, consider the most typical situation in which the elements are chosen as $d$-dimensional simplexes (i.e., intervals when $d=1$, triangles when $d=2$, or tetrahedrons when $d=3$ ) and the basis functions $\phi_{j}(\mathbf{p})$ are piecewise linear. Further, let $\mathcal{E}_{1}, \ldots, \mathcal{E}_{v}$ denote the elements of the considered mesh and, for a generic element $\mathcal{E}_{j}$, let $\mathcal{V}_{j} \subset\{1, \ldots, n\}$ be the set of indices corresponding to the vertices of $\mathcal{E}_{j}$. Notice that, for a $d$-dimensional simplex, $\mathcal{V}_{j}$ contains exactly $d+1$ elements. Then, in this case, the input matrix $\mathbf{B}\left(\mathbf{p}^{0}\right)$ can be written as a convex combination of the input matrices associated to the vertices of the element $\mathcal{E}_{j}$ containing $\mathbf{p}^{0}$, i.e.,

$$
\mathbf{B}\left(\mathbf{p}^{0}\right)=\sum_{i \in \mathcal{V}_{j}} \omega_{i}\left(\mathbf{p}^{0}\right) \mathbf{B}\left(\mathbf{p}_{i}\right)
$$

where $\omega_{i}\left(\mathbf{p}^{0}\right) \geq 0$ and $\sum_{i \in \mathcal{V}_{j}} \omega_{i}\left(\mathbf{p}^{0}\right)=1$. This makes it possible to derive sufficient conditions for source identifiability which has to be checked only for each pair of elements. To this end, for a generic element $\mathcal{E}_{j}$, let $\mathbf{B}\left(\mathcal{E}_{j}\right)$ denote the matrix obtained by row juxtaposition of the column matrices $\mathbf{B}\left(\mathbf{p}_{i}\right)$ with $i \in \mathcal{V}_{j}$, and let $\mathbf{B}_{1}\left(\mathcal{E}_{j}\right)$ be obtained in an analogous way from the matrices $\mathbf{B}_{1}\left(\mathbf{p}_{i}\right)$ with $i \in \mathcal{V}_{j}$. Then, the following result holds.

Theorem 2: Let the input matrices be as in (12) and, for any pair of distinct elements $\mathcal{E}_{j}, \mathcal{E}_{\ell}$ of the mesh, consider the polynomial matrix

$$
\boldsymbol{\Psi}_{j \ell}(z)=\left[\begin{array}{ccc}
z \mathbf{I}-\mathbf{A}_{11} & \mathbf{B}_{1}\left(\mathcal{E}_{j}\right) & \mathbf{B}_{1}\left(\mathcal{E}_{\ell}\right) \\
\mathbf{C}_{1} & \mathbf{0} & \mathbf{0}
\end{array}\right]
$$

with $z \in \mathbb{C}$. Then, the following facts hold:

(i) in case (a), the source is identifiable if

$$
\operatorname{rank}\left\{\boldsymbol{\Psi}_{j \ell}(z)\right\}=n_{o}+\left|\mathcal{V}_{j} \cup \mathcal{V}_{\ell}\right|
$$

for any $z \in \mathbb{C}$ and for any $\mathcal{E}_{j}, \mathcal{E}_{\ell}$ with $j \neq \ell$;

(ii) in case (b), the source is identifiable if the rank condition (13) holds for any $z \in \operatorname{sp}\{\mathbf{F}\}$ and for any $\mathcal{E}_{j}, \mathcal{E}_{\ell}$ with $j \neq \ell$.

Notice that in condition (13), the term $\left|\mathcal{V}_{j} \cup \mathcal{V}_{\ell}\right|$ represents the number of distinct vertices in $\mathcal{E}_{j} \cup \mathcal{E}_{\ell}$. As a consequence, $\left|\mathcal{V}_{j} \cup \mathcal{V}_{\ell}\right| \leq 2(d+1)$ where the equality holds if and only if $\mathcal{E}_{j}$ and $\mathcal{E}_{\ell}$ have no common vertices. Hence, Theorem 2 suggests that, in the $d$-dimensional case, $2(d+1)$ sensors may be needed in order to have source identifiability.

Remark 1: The rank condition of Theorem 2 provides a computationally feasible way to verify whether a given set of sensors guarantees source identifiability, since it has to be checked only for a finite number of cases, i.e., for each pair of distinct elements. Additional insights can be gained by recalling that system (8) can also be written as a linear descriptor system [see (6)]

$$
\begin{aligned}
\mathbf{E x}_{k+1} & =\mathbf{M} \mathbf{x}_{k}+\delta t \boldsymbol{\phi}\left(\mathbf{p}^{0}\right) u_{k} \\
\mathbf{y}_{k} & =\mathbf{C} \mathbf{x}_{k}
\end{aligned}
$$

where the matrices $\mathbf{E}, \mathbf{M}$ and $\phi\left(\mathbf{p}^{0}\right)$ have very specific structures. In particular, the non-zero elements of the matrices $\mathbf{E}, \mathbf{M}$ correspond to connected vertices in the graph associated to the FE mesh. Further, for any mesh vertex $\mathbf{p}_{i}$, $\phi\left(\mathbf{p}_{i}\right)$ coincides with $\mathbf{e}_{i}$, the $i$-th vector of the canonical basis. If it is assumed that system (14) is observable, it can be easily shown that in condition (13) the matrix $\boldsymbol{\Psi}_{j \ell}(z)$ can be replaced by

$$
\tilde{\boldsymbol{\Psi}}_{j \ell}(z)=\left[\begin{array}{ccc}
z \mathbf{E}-\mathbf{M} & \phi\left(\mathcal{E}_{j}\right) & \phi\left(\mathcal{E}_{\ell}\right) \\
\mathbf{C} & \mathbf{0} & \mathbf{0}
\end{array}\right]
$$

where $\phi\left(\mathcal{E}_{j}\right)$ denotes the matrix obtained by row juxtaposition of the column matrices $\phi\left(\mathbf{p}_{i}\right)=\mathbf{e}_{i}$ with $i \in \mathcal{V}_{j}$. Hence, if it is further assumed that the sensor locations coincide with vertices of the FE mesh, it is possible to relate the rank of the matrix $\tilde{\boldsymbol{\Psi}}_{j \ell}(z)$ to the topology of the FE mesh as well as to the sensor locations. In fact, results on the rank of matrices of the form of $\tilde{\boldsymbol{\Psi}}_{j \ell}(z)$ for systems like (14) defined over graphs have been recently obtained in the literature [17]. While such results are only generic (i.e., they hold for almost all the dynamical systems compatible with the graph topology, but counterexamples can exist), nevertheless they provide useful guidelines on where to place the sensors inside the domain $\Omega$. The interested reader is referred to [17] for further details on this issue.

\section{SOURCE ESTIMATION}

Based on the fact that distinct source locations correspond to different process behaviors, and thanks to finite element approximation, the key idea of the proposed source estimation algorithms relies upon the assumption that system (7), at each time step, obeys to one of a finite set of diffusion models. To this end, the Multiple Model (MM) approach [18] provides a suitable tool, as it accounts for the uncertainty about the system input location, assuming that the real evolution of the system follows one of the possible modes of operation. In particular, the idea is to match each hypothesis of source being located in a generic element of the mesh, to a distinct operating mode of the system. This makes it possible to run in parallel a finite number of mode-matched Kalman filters, one for each element of the generated mesh. Hence, each mode $m^{j}$ associated to the hypothesis that a point source is located in $\mathbf{p}^{0}$, contained in element $\mathcal{E}_{j}$, is characterised by the following mode-matched model

$$
\begin{aligned}
\mathbf{x}_{k+1} & =\mathbf{A} \mathbf{x}_{k}+\mathbf{B}\left(\mathcal{E}_{j}\right) \boldsymbol{\omega}\left(\mathbf{p}^{0}\right) u_{k}+\mathbf{w}_{k}, \quad j=1,2, \ldots, v \\
\mathbf{y}_{k} & =\mathbf{C} \mathbf{x}_{k}+\mathbf{v}_{k}
\end{aligned}
$$

where $\boldsymbol{\omega}\left(\mathbf{p}^{0}\right)$ is the $(d+1)$-dimensional column vector of coefficients $\omega_{i}\left(\mathbf{p}^{0}\right), i \in \mathcal{V}_{j}$, introduced in section IV. It is worth noting that in order to be able to detect new sources, an extra source-free operating mode, based on the assumption that no point source is present, needs to be added to the set of possible modes of the MM algorithm. Thus, including the no-source mode, from now on denoted as $m^{r}$ for $r=v+1$, and recalling the mesh generates elements $\mathcal{E}_{1}, \ldots, \mathcal{E}_{v}$, the set of possible modes becomes $r$-dimensional.

Bearing in mind the previous points about source detection and localisation, the additional joint source intensity and state estimation can be carried out by constructing an augmented system for the MM estimator, as the aggregate of the 
original system (15) and a suitable model for the unknown input time evolution. To this end, let us introduce $\boldsymbol{\eta}_{k}=$ $\operatorname{col}\left(\omega_{i}\left(\mathbf{p}^{0}\right) u_{k}, i \in \mathcal{V}_{j}\right)$ so that $\mathbf{B}\left(\mathbf{p}^{0}\right) u_{k}=\mathbf{B}\left(\mathcal{E}_{j}\right) \boldsymbol{\eta}_{k}$. Then, the augmented system for a generic mode $m^{j}$ originated from (15), takes the following form for $j=1,2, \ldots, v$

$$
\begin{aligned}
{\left[\begin{array}{l}
\mathbf{x}_{k+1} \\
\boldsymbol{\eta}_{k+1}
\end{array}\right] } & =\left[\begin{array}{cc}
\mathbf{A} & \mathbf{B}\left(\mathcal{E}_{j}\right) \\
\mathbf{0} & \mathbf{I}
\end{array}\right]\left[\begin{array}{l}
\mathbf{x}_{k} \\
\boldsymbol{\eta}_{k}
\end{array}\right]+\left[\begin{array}{c}
\mathbf{w}_{k} \\
\boldsymbol{\zeta}_{k}
\end{array}\right] \\
\mathbf{y}_{k} & =\left[\begin{array}{ll}
\mathbf{C} & \mathbf{0}
\end{array}\right]\left[\begin{array}{l}
\mathbf{x}_{k} \\
\boldsymbol{\eta}_{k}
\end{array}\right]+\mathbf{v}_{k}
\end{aligned}
$$

whereas, for $j=r$

$$
\begin{aligned}
\mathbf{x}_{k+1} & =\mathbf{A} \mathbf{x}_{k}+\mathbf{w}_{k} \\
\mathbf{y}_{k} & =\mathbf{C} \mathbf{x}_{k}+\mathbf{v}_{k}
\end{aligned}
$$

Note that in (16) the dynamics of the source intensity $u_{k}$ are assumed to follow a discrete-time random walk. As a result, the joint source and field estimation problem can be reduced to the joint estimation of $\mathbf{x}_{k}$ and $\boldsymbol{\eta}_{k}$ for each time step $k$. Assuming the source may move within the domain, i.e. the correct operating mode may switch over time, it is convenient to employ a dynamic MM technique, e.g. Interacting Multiple Model (IMM) [18], which allows for mode jumps, limiting at the same time the number of hypotheses to the number of filters. Otherwise, the source of interest is assumed motionless, i.e. fixed in an unknown position of the monitored area. In this case a static MM estimator, which assumes there is a single operating mode throughout the entire process, can be suitably employed to address the considered estimation problem. Next, a brief summary of a centralised approach to the static MM called Finite Element Static Multiple Model (FE-SMM) and dynamic FE-IMM is shown, wherein the measurements of all sensors are collected and jointly processed in the correction step of each modematched filter. For further details on multiple-model filtering algorithms, the reader is referred to [18].

\section{A. Static case: FE-SMM}

The FE-SMM algorithm runs a bank of $r$ FE Kalman filters matched to the modes $m^{j}$ in (16), for $1 \leq j<r$, or (17) for $j=r$. Each filter updates the state estimate, covariance and mode probability relative to mode $m^{j}$ by processing the entire set of gathered measurements $y_{k, i}, i \in \mathcal{S}$. Once initialised, mode-matched filters run independently with no interaction. At the end of each cycle, the mode with maximum probability will be considered as the operating one. As a consequence, the associated mode-conditioned estimate will be directly used for field and source intensity estimation. Further, exploiting the structure of the FE approximation, the source location can be estimated as a convex combination of the position of the vertices of the element $\mathcal{E}_{j}$ matched to the estimated operating mode, i.e.

$$
\begin{aligned}
\hat{\mathbf{p}}^{0} & =\sum_{i \in \mathcal{V}_{j}} \hat{\omega}_{i} \mathbf{p}_{i} \\
\hat{\omega}_{i} & =\frac{\hat{\eta}_{k}^{i}}{\hat{u}_{k}}, \quad i \in \mathcal{V}_{j}, \quad \hat{u}_{k}=\sum_{i \in \mathcal{V}_{j}} \hat{\eta}_{k}^{i}
\end{aligned}
$$

\section{B. Dynamic case: FE-IMM}

The idea is to run an IMM estimator for the augmented system (16) with mode-to-mode transitions modelled by means of a homogeneous Markov chain with known constant transition probabilities $\pi_{i j}=$ $\operatorname{prob}\left(m_{k}=m^{i} \mid m_{k-1}=m^{j}\right), i, j \in\{1,2, \ldots, r\}$ where $m_{k} \in\left\{m^{j}\right\}_{j=1}^{r}$ represents the modal state (i.e. the mode in operation) at time $k$. Differently from the static MM algorithm, at the beginning of each sampling interval, the $r$ filters interact in a mixing step which produces the so called mixed initial conditions, i.e. different combinations of the previous model-conditioned estimates and associated covariances. It must also be noted that, since the source-free mode $m^{r}$ has a different (lower) state dimension with respect to modes $m^{j}, j \neq r$, the state estimate and covariance of the former must be padded with zeros in order to match the higher dimension of the latter during the mixing step.

The proposed source estimator uses $v+1$ Kalman filters, where $v$ is the number of elements of the FE mesh. More precisely, $v$ out of the $v+1$ Kalman filters have an $(n+d+1)$ dimensional state, where $n$ is the number of vertices of the FE grid and $d \in\{1,2,3\}$ the dimension of the domain of interest, while the remaining Kalman filter associated to the no-source mode has $n$-dimensional state. Since $v=O(n)$, $d \ll n$ and $|\mathcal{S}| \ll n$, each Kalman filter has $O\left(n^{3}\right)$ complexity, and the overall computational complexity is $O\left(n^{4}\right)$. It is also worth pointing out that the modal Kalman filters can be run in a fully parallel fashion, being mindful however, that the IMM mixing step requires an exchange of information between the bank of filters.

\section{NUMERICAL EXAMPLES}

The proposed FE-IMM, described in Section V-B, is validated via simulations. Consider a scenario concerning a moving source estimation for a diffusion process governed by the 2D case of (1) with $\mathcal{A}(x)=-\lambda\left(\partial^{2} x / \partial \xi^{2}+\partial^{2} x / \partial \eta^{2}\right)$ and mixed boundary conditions

$$
\begin{array}{rll}
\partial x / \partial \mathbf{n}+\beta_{1} x & =g_{1} & \text { on } \partial \Omega_{1} \\
\partial x / \partial \mathbf{n} & =0 & \text { on } \partial \Omega_{2} ;
\end{array}
$$

This model describes, for instance, transient contaminant transport in water bodies. Parameters $\beta_{1}=\frac{\nu}{\lambda}$ and $g_{1}=\frac{\nu}{\lambda} x_{e}$ are such that (19) describes an outward/inward diffusive flux across $\partial \Omega_{1}$ (boundary 10 in Fig.1), proportional to the concentration difference $x-x_{e}$ between internal and external environments (external concentration $x_{e}=0$ is assumed known, $\nu=1$ ). The homogeneous Neumann boundary condition (20) assumes there is no flux across $\partial \Omega_{2}$, i.e. it is considered impermeable to the contaminant. Further, (1) implicitly assumes $\lambda$ is constant, here taken as $\lambda=0.1$. A network of 6 sensors is randomly deployed inside the spatial 2D domain $\Omega$ to sample the concentration field of interest, with sampling interval $T_{s}=1[s]$ and standard deviation of measurement noise $\sigma_{v}=0.005$. As shown in Fig. 1, a triangular mesh (116 nodes, 196 elements) is generated over $\Omega$ for the finite-dimensional approximation of the monitored 


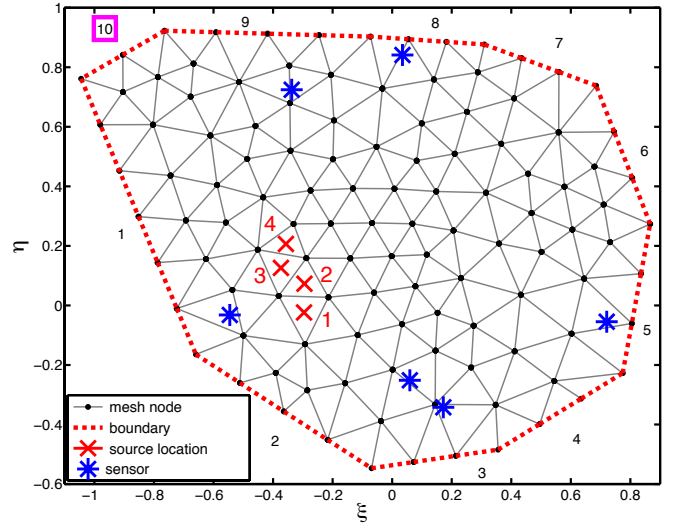

Fig. 1: Source moves from 1 to 4 in the monitored area $\Omega$.
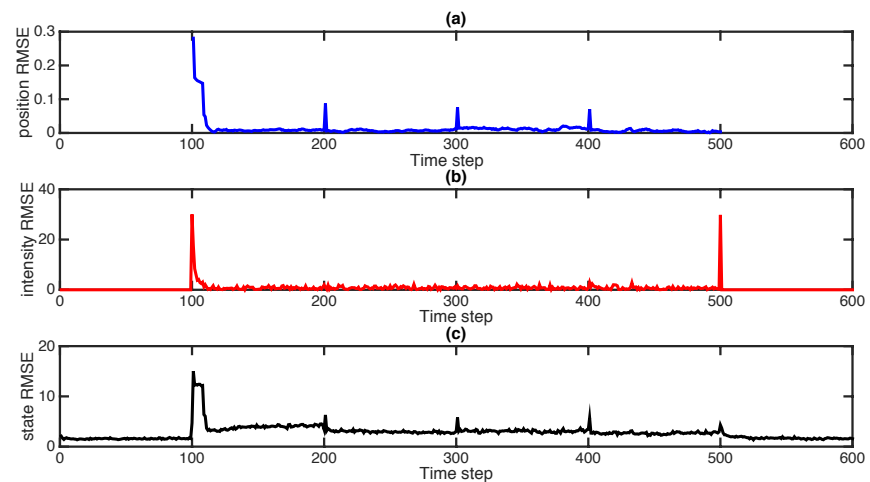

Fig. 2: Simulation results in the case of dynamic source.

field. As true initial field condition, we consider $\mathbf{x}_{0}=$ $\mathbf{0}$, whereas the estimator starts from $\hat{\mathbf{x}}_{1 \mid 0}=10 \cdot \mathbf{1}$ with covariance $\mathbf{P}_{1 \mid 0}=100^{2} \mathbf{I}$. Moreover, the source intensity estimate is initialised as $\hat{u}_{1 \mid 0}=10$, with associated initial covariance matrix $\mathbf{P}_{1 \mid 0}^{u}=100^{2} \mathbf{I}$, while the true average intensity of the source is 30 . The standard deviation of the process noise for the simulator is set as $\sigma_{w}=1.5$, whereas for the MM filters $\hat{\sigma}_{w}=5$. The variance of the disturbance input is set to $\sigma_{\zeta}^{2}=0.04$. All simulation results are averaged over 100 Monte Carlo trials.

The source, activated at time $100[s]$, is moving along the path $1-2-3-4$ (see Fig. 1), sojourning 100 time steps in each intermediate location, before turning off at time 500. From 500 to 600 the simulation continues with no source. Jump probabilities are $\pi_{i i}=0.85 \forall i=1, \ldots, r$, $\pi_{i r}=0.05, i=1, \ldots, v$, while the remaining 0.1 probability is equally distributed among all elements $\mathcal{E}_{j}$ adjacent to $\mathcal{E}_{i}$.

The Root Mean Square Errors (RMSE) relative to the source position, intensity and source-induced field, are reported, as performance indices, in Fig. 2. These are obtained via comparison of the estimated quantities with a simulated system (ground truth), which implements a finer mesh (427 nodes, 784 elements) and runs at a higher sampling frequency of $10 \mathrm{~Hz}$, in order to take into account model uncertainty. Results show that both the estimators succeed in localizing the unknown source (Fig. 2a) and estimating the corresponding intensity (Fig. 2b) in a very short time and with remarkable accuracy. The field estimation error (Fig. 2c) presents visible peaks in correspondence of either source activation or change of position, but it promptly stabilizes once the input has been detected.

\section{CONCLUSIONS}

The paper has addressed the problem of detecting a diffusive point source and jointly estimating its location, intensity and induced field from pointwise-in-time-and-space field measurements of sensors deployed over the monitored area. This has been made possible by combining the finite-element method for discretising in space the diffusion dynamics and the multiple-model Kalman filtering approach. Future work will concern the multi-source case.

\section{REFERENCES}

[1] T. Zhao and A. Nehorai, "Distributed sequential Bayesian estimation of a diffusive source in wireless sensor networks", IEEE Transactions on Signal Processing, vol. 55, no. 4, pp. 1511-1524, 2007.

[2] B. Ristic, A. Gunatilaka, and R. Gailis, "Achievable accuracy in Gaussian plume parameter estimation using a network of binary sensors", Information Fusion, vol. 25, pp. 42-48, 2015.

[3] T. van Waterschoot and G. Leus, "Distributed estimation of static fields in wireless sensor networks using the finite element method", IEEE Int. Conf. on Acoustics, Speech and Signal Processing (ICASSP) pp. 2853-2856, 2012.

[4] J. Weimer, B. Sinopoli, and B.H. Krogh, "Multiple source detection and localization in advection-diffusion processes using wireless sensor networks", 30th IEEE Real-Time Systems Symposium, RTSS 2009, pp. 333-342, 2009.

[5] M.A. Demetriou, "Process estimation and moving source detection in 2-D diffusion processes by scheduling of sensor networks", American Control Conference, ACC, pp. 3432-3437, 2007.

[6] S.S. Ram and V.V. Veeravalli, "Localization and intensity tracking of diffusing point sources using sensor networks", IEEE Global Telecomm. Conf., GLOBECOM '07, pp. 3107-3111, 2007.

[7] L.A. Rossi, B. Krishnamachari, and C.-C.J. Kuo, "Distributed parameter estimation for monitoring diffusion phenomena using physical models", IEEE Conf. Sensor and Ad Hoc Comm. and Networks, IEEE SECON 2004, pp. 460-469, 2004.

[8] R. Bellman and K.J. Åström, "On structural identifiability", Mathematical Biosciences, vol. 7, pp. 329-339, 1970.

[9] J.A. Jacquez and P. Greif, "Numerical parameter identifiability and estimability: Integrating identifiability, estimability, and optimal sampling design”, Mathematical Biosciences, vol. 77, pp. 201-227, 1985.

[10] A. El Badia, T. Ha-Duong, and A. Hamdi, "Identification of a point source in a linear advection-dispersion-reaction equation: Application to a pollution source problem", Inverse Problems, vol. 21, pp. 11211136, 2005

[11] G. Pelosi, R. Coccioli, and S. Selleri, Quick finite elements for electromagnetic waves, Artech House, Norwood, MA, 2009.

[12] S.C. Brenner and L.R. Scott, The mathematical theory of finite element methods, Springer-Verlag, New York, NY, 1996.

[13] P.P. Silvester and R. Ferrari, Finite Elements for Electrical Engineers, Cambridge University Press, Cambridge, UK, 3rd ed., 1996.

[14] R. Becker and R. Rannacher, "An optimal control approach to a posteriori error estimation in finite element methods", Acta Numerica, pp. 1-101, Cambridge University Press, 2001.

[15] S. Gillijns and B. De Moor, "Unbiased minimum-variance input and state estimation for linear discrete-time systems", Automatica, vol. 43, no. 1, pp. 111-116, 2007.

[16] B. Li, "State estimation with partially observed inputs: A unified Kalman filtering approach", Automatica, vol. 49, no. 3, pp. 816-820, 2013.

[17] F. Pasqualetti, F. Dorfler, and F. Bullo, "Attack detection and identification in cyber-physical systems," IEEE Transactions on Automatic Control, vol. 58, no. 11, pp. 2715-2729, 2013.

[18] Y. Bar-Shalom, X.R. Li and T. Kirubarajan. Estimation with Applications to Tracking and Navigation: Theory Algorithms and Software. Wiley, 2004. 